\title{
Follow-up of Gravitational Wave Events with the Fermi-LAT. Status and Prospects for the Future
}

\author{
Nicola Omodei*广 \\ W. W. Hansen Experimental Physics Laboratory, Kavli Institute for Particle Astrophysics and \\ Cosmology, Department of Physics and SLAC National Accelerator Laboratory, Stanford \\ University, Stanford, CA 94305, USA \\ E-mail: nicola.omodei@stanford.edu
}

\section{Giacomo Vianello}

W. W. Hansen Experimental Physics Laboratory, Kavli Institute for Particle Astrophysics and Cosmology, Department of Physics and SLAC National Accelerator Laboratory, Stanford University, Stanford, CA 94305, USA

\section{Daniel Kocevski}

Astrophysics Office, ST12, NASA/Marshall Space Flight Center, Huntsville, AL 35812, USA

\section{Sara Buson}

NASA Goddard Space Flight Center, Greenbelt, MD 20771, USA

\section{Niccolò Di Lalla}

Università di Pisa and Istituto Nazionale di Fisica Nucleare, Sezione di Pisa I-56127 Pisa, Italy

\begin{abstract}
As the first detections of Gravitational Waves (GW) from the coalescence of compact objects were announced by LIGO and Virgo, a new era for astronomy began. Searches for electromagnetic (EM) counterparts of GW events are of fundamental importance, as their success will increase the confidence in the GW detection and will help characterize the system parameters. The Fermi Gamma-ray Space Telescope is the most capable observatory to simultaneously observe a large fraction of the sky from $10 \mathrm{keV}$ to more than $300 \mathrm{GeV}$, providing the unique capability of rapidly covering the entire probability region from a LIGO candidate. In this paper, we will present the strategy for follow-up observations of GW events with the Fermi Large Area Telescope (LAT), focusing on the results from the first science runs $\mathrm{O} 1 / \mathrm{O} 2$. We will also discuss the prospects for detections of GW in coincidence with a gamma-ray signal from the Fermi Gamma-ray Burst Monitor (GBM) and the LAT, likely from a short Gamma-Ray Burst (sGRB) arising from the merger of two neutron stars.
\end{abstract}

7th Fermi Symposium 2017

15-20 October 2017

Garmisch-Partenkirchen, Germany

\footnotetext{
* Speaker.

${ }^{\dagger}$ on behalf of the Fermi-LAT collaboration
} 


\section{A new era}

The detection of gravitational waves (GWs) has opened a new window to the cosmos and paved the way to a new era of multi-messenger astronomy. The first detected signals by Advanced Laser Interferometer Gravitational-Wave Observatory (LIGO) (1), GW 150914 and GW 151226 (2; $3 ; 4 ; 5)$ are compatible with the coalescence of high-mass binary black holes (BBHs). The detection of a third BBH merger, GW 170104 (6) adds to the growing sample BBH merger events. The first detection of a binary neutron star system, GW 170817 (7) followed by the detection of a short GRB (SGRB) by the Fermi Gamma-ray Burst Monitor (GBM) $(8$; 9) and by INTEGRAL SPI-ACS $(10 ; 11)$ was the confirmation of the long suspected connection between SGRBs and compact binary coalescence. The Fermi Large Area Telescope (LAT, 12) has a lower detection rate with respect to the GBM, but can provide $0.2-0.3^{\circ}$ localizations. In the case of a detection of an electromagnetic (EM) counterpart, the LAT could substantially reduce the localization uncertainty, facilitating follow-up at other wavelengths. The two instruments on-board the Fermi satellite are complementary and uniquely capable of providing all-sky observations from hard X-rays to high-energy gamma rays in normal survey operations. Together, they cover the entire localization probability maps of gravitational wave events within hours $(\sim 6)$ of their detections. Our searching strategy relies on automated processing of the data, which is very important to rapidly alert the community in case of a detection with the goal of providing reliable and accurate localization of the EM counterpart and narrow down the searching radius. To this end, we set up pipelines that automatically download the data from the Fermi-LAT dataserver ${ }^{1}$, downsample the LIGO/Virgo credibility map to roughly match the LAT Point Spread Function $\left(\sim 4^{\circ}\right.$ at $\left.100 \mathrm{MeV}\right)$, and compute unbinned likelihood analysis in each pixel. Details of this technique are described in Vianello et al., 2017 (13) while the results on GW 150914, GW 151226 and LV 151012, and GW 170104 are presented in $(14 ; 15 ; 16)$ respectively.

\section{Fermi LAT observation of GW 170817}

The GW 170817 event was in an unlucky position for the Fermi-LAT, as the satellite was entering the SAA at the time of the LIGO/Virgo trigger ( $t_{\mathrm{GW}}=2017-08-17$ 12:41:04.444 UTC). During SAA passages the LAT and the GBM do not collect data due to the high charged particle background in this region. Because of the higher susceptibility of the LAT to the charged particles in this region, the SAA boundary employed by the LAT encompasses a $\sim 14 \%$ larger area than the boundary used by the GBM, resulting in slightly different times at which the two instruments do not collect data. GBM triggered on GRB170817A at $\mathrm{t}_{\mathrm{EM}}=2017-08-17$ 12:41:06.475 UTC. The LAT data taking was switched off approximately one minute before $t_{\mathrm{EM}}$ while the GBM switched off approximately two minutes after $t_{\mathrm{EM}}$. The LAT resumed data taking upon exiting the SAA at $\mathrm{t}_{\mathrm{GW}}+1153 \mathrm{~s}$. At that time, the entire $90 \%$ credible region of the LAL Inference map was within the LAT FoV and the region subsequently exited at $t_{\mathrm{GW}}+2027 \mathrm{~s}$. At the position of the optical counterpart $(17 ; 18)$ the value for the flux upper bound over this interval and in the $0.1-1 \mathrm{GeV}$ energy range is $4.5 \times 10^{-10} \mathrm{erg} \mathrm{cm}^{-2} \mathrm{~s}^{-1}$ (95\% confidence level).

\footnotetext{
${ }^{1}$ https://fermi.gsfc.nasa.gov/cgi-bin/ssc/LAT/LATDataQuery.cgi
} 
Considering the proximity of the source $(42.9 \pm 3.2$ $\mathrm{Mpc}$ (19) this value corresponds to an equivalent isotropic luminosity of approximately $9.7 \times 10^{43} \mathrm{erg}$ $\mathrm{s}^{-1}$, which being so low compared to other Fermi-LAT detected GRBs with known redshift, is extremely constraining (Fig. 1). We can rule out with high confidence a late-time emission of GRB170817A as luminous as other Fermi-LAT bursts. For typical on axis GRBs, the afterglow is coincident with the end of the prompt emission $(20 ; 21)$, while for off-axis jets, the onset of the afterglows is predicted to be of the order of few days up to 100 days $(22 ; 23)$. As reported in (24) and (25), an X-ray source positionally coincident with the optical transient was detected at $\sim 9$ days after the GW event, followed by a radio source detection (26) suggesting the detection of an afterglow from a possible off-axis jet (18). We monitored the source by performing a likelihood analysis in every interval of time after the trigger when the source was in the LAT field of view. In a time period spanning from $\mathrm{t}_{\mathrm{EM}}-1$ day to $\mathrm{t}_{\mathrm{EM}}+45$ days the values of the flux upper bound range between $9.7 \times 10^{-11}$ to $3.7 \times 10^{-8} \mathrm{erg} \mathrm{cm}^{-2} \mathrm{~s}^{-1}$

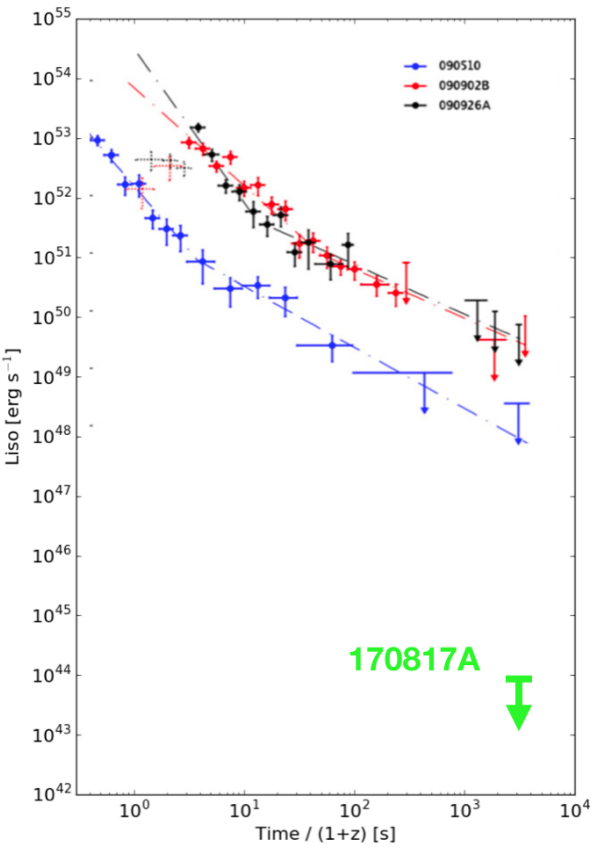

Figure 1: Comparison of the luminosity upper bound of GRB 170817A (green arrow) with other three LAT-detected GRBs with known redshift.

corresponding to a luminosity range of $2.1 \times 10^{43}$ to $8.1 \times 10^{45} \mathrm{erg} \mathrm{s}^{-1}(0.1-1 \mathrm{GeV})$. Integrating the data on the life time of the mission ( 9 years), we also obtain a flux upper bound of $\mathrm{F}<1.32 \times 10^{-12}$



\section{Looking ahead}

We characterize here the sensitivity of the LAT to SGRBs in general. As we describe in detail in (27), we simulate a transient point source with a spectrum $d N / d E \propto E^{-2}$, fading rapidly as $\propto T^{-1}(28)$, and vary the integrated flux until $50 \%$ of the realizations of the simulated source are detected above $5 \sigma$. The starting time of a GRB observation is critical: we can detect fainter SGRBs the earlier we start observing. In Fig. 2 we show how the sensitivity of the LAT for sources at midGalactic latitudes changes for five different starting times. The first four shaded regions are for observations with a duration of $100 \mathrm{~s}$, starting respectively at $T_{0}, T_{0}+2 \mathrm{~s}, T_{0}+10 \mathrm{~s}, T_{0}+100 \mathrm{~s}$, while the last is between $T_{0}+1153 \mathrm{~s}$ and $T_{0}+2027 \mathrm{~s}$ after the trigger time as for GRB 170817A. For reference we also report the measurements for other SGRBs detected by the LAT, as well as the upper bound for GRB 170817A in the $100 \mathrm{MeV}-100 \mathrm{GeV}$ energy range. Among the sample of SGRBs detected by the LAT, we note that the fluences of GRB 081024B and GRB 140402A measured by the GBM in the $10 \mathrm{keV}-1 \mathrm{MeV}$ energy band are similar to the one of GRB 170817A. GRB 090510 is the brightest SGRB detected by the LAT so far and resulted in the detection of both its prompt and extended emission. The much dimmer GRB 130804A, on the other hand, was in the 
field of view at the time of the trigger, but was only detected by the LAT at $T_{0} \sim 200 \mathrm{~s}$, constituting an example of delayed high-energy emission.

The statistics are limited, but we can conclude that the LAT needs to start observing a source within $100-200 \mathrm{~s}$ to have a chance at detecting bursts as luminous as the brightest of the LAT-detected SGRBs. The LAT detection efficiency for short GRBs (SGRBs) decreases significantly after 100200 s. The Fermi-GBM observes $\sim 65 \%$ of the sky, with the rest being occulted by the Earth. In survey strategy, the LAT observes $\sim 35 \%$ of SGRBs detected by the GBM within $\sim 100 \mathrm{~s}$ of the trigger, which translates in $\sim 23 \%$ of the full-sky SGRB population being observed (either detected or not) within $100 \mathrm{~s}$ from their GBM trigger. The LAT detects $\sim 5 \%$ of all GBMdetected SGRBs. If we assume the LAT will have the same efficiency for GRB/GW triggers and a rate of joint GBM/GW events of 1 (2) per year, we obtain at most a 5\% ( $\sim 10 \%$ ) probability of detecting one or more GRB/GW with the LAT in one year, respectively. Currently the Fermi spacecraft autonomously slews to bring GRBs within the LAT field of view only when the GBM de-

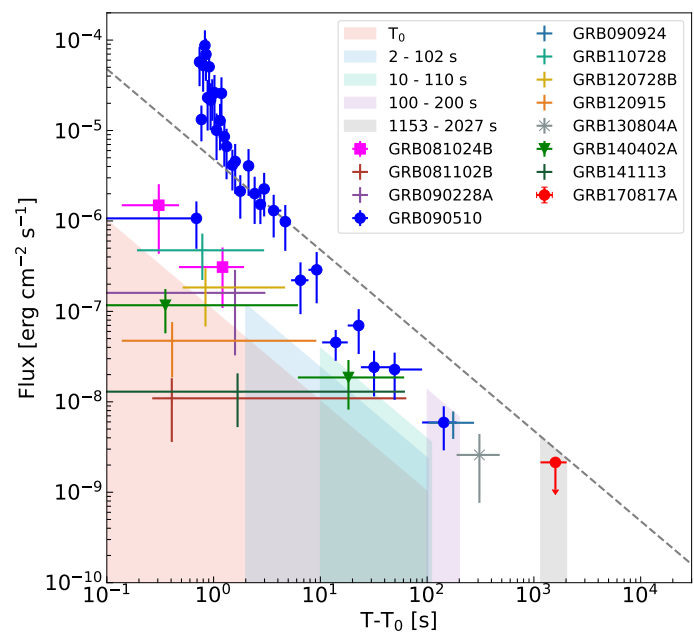

Figure 2: Light curves of the LAT-detected SGRBs. We highlight GRB 081024B (magenta squares), GRB 140402A (green triangles) GRB 130804 (gray cross), GRB 090510 (blue circles) and the fluence upper bound of GRB 170817A at the time of the first LAT observation (red circle). The shaded boxes represent the sensitivity to simulated sources detected with TS $>25,50 \%$ of the time for observations starting at different times (see legend). The sensitivity curve for an observation between 1153 and $2027 \mathrm{~s}$, as for GRB $170817 \mathrm{~A}$ is also extrapolated back in time according to a $T^{-1}$ decay law (dashed gray line). tects bursts of exceptionally high-peak flux.

A modification of this strategy to repoint to lower-fluence SGRBs would provide increased exposure to dimmer events like GRB 170817A and increase the chances of detecting long-lived afterglow emission from such sources. Simulations have shown that this would allow the LAT to observe $35 \%$ of SGRBs within $100 \mathrm{~s}$, enhancing the probability of detecting one or more GRB/GW events per year to $\sim 7 \%(\sim 13 \%)$ for a GBM/GW rate of 1 (2) per year.

\section{Conclusions}

A new era for Multi Messenger Astronomy has officially begun with the discovery of a gravitation wave signal and an Electromagnetic signal originating from the same astronomical source. The discovery of GW 170817/GRB 170817A strongly supports the conjectured association of SGRBs with merging neutron stars. Fermi-LAT was already switched off due to SAA encounter at the time of the GW/GBM trigger, therefore it is only possible to set upper bounds at approximately 1000 seconds after the trigger, when the location of the source entered the LAT FoV. Due to the proximity of the source, the upper bound is very constraining, ruling out long-lasting emission of 
the same luminosity of other LAT-detected GRBs. Continuous monitoring is ongoing, since we cannot exclude a priori very late time emission from this peculiar object. The probability to detect with the LAT the high-energy gamma-ray counterpart of a GW event is approximately 5\% (10\%) with an estimated rate or 1 (2) merger events per year. This is compatible with the detection of one (few) events in the next few years making a strong case for the extension of the Fermi mission. If the LAT detects an EM signal from a GW source we will provide a good localization (of the order of $0.2^{\circ}$ ) and, as in the case of other published LAT detected bursts, will be able to carefully study the system at high energy, constraining its overall energetics, studying the jet structure, its Lorentz factor and viewing angle (28). Even in the case of a negative detection an upper bound placed during the prompt emission would place a very strong limit on the emitting scenarios for this class of objects. From what we know from GBM and LAT: there is every reason to believe that gamma-ray observations will play a crucial rule in the developments of the exciting field of multi-messenger astronomy.

\section{Acknowledgments}

The Fermi-LAT Collaboration acknowledges support for LAT development, operation and data analysis from NASA and DOE (United States), CEA/Irfu and IN2P3/CNRS (France), ASI and INFN (Italy), MEXT, KEK, and JAXA (Japan), and the K.A. Wallenberg Foundation, the Swedish Research Council and the National Space Board (Sweden). Science analysis support in the operations phase from INAF (Italy) and CNES (France) is also gratefully acknowledged. This work performed in part under DOE Contract DE-AC02-76SF00515.

\section{References}

[1] Ligo Scientific Collaboration and Virgo Collaboration collaboration, B. P. Abbott, R. Abbott, T. D. Abbott, M. R. Abernathy, F. Acernese, K. Ackley et al., Gw150914: The advanced ligo detectors in the era of first discoveries, Phys. Rev. Lett. 116 (Mar, 2016) 131103.

[2] B. P. Abbott, R. Abbott, T. D. Abbott, M. R. Abernathy, F. Acernese, K. Ackley et al., Localization and Broadband Follow-up of the Gravitational-wave Transient GW150914, ApJ 826 (July, 2016) L13, [1602.08492].

[3] Ligo Scientific Collaboration and Virgo Collaboration collaboration, B. P. Abbott, R. Abbott, T. D. Abbott, M. R. Abernathy, F. Acernese, K. Ackley et al., Properties of the binary black hole merger gw150914, Phys. Rev. Lett. 116 (Jun, 2016) 241102.

[4] Virgo, LIGO SCIENTIFIC collaboration, B. P. Abbott et al., Binary Black Hole Mergers in the first Advanced LIGO Observing Run, Phys. Rev. X 6 (2016) 041015, [1606.04856].

[5] VIRGO, LIGO ScIENTIFIC collaboration, B. P. Abbott et al., GW151226: Observation of Gravitational Waves from a 22-Solar-Mass Binary Black Hole Coalescence, Phys. Rev. Lett. 116 (2016) 241103, [160 6.04855 ].

[6] VIRGO, LIGO SCIENTIFIC collaboration, B. P. Abbott et al., GW170104: Observation of a 50-Solar-Mass Binary Black Hole Coalescence at Redshift 0.2, Phys. Rev. Lett. 118 (2017) 221101, [1706.01812].

[7] Virgo, LIGO SCIENTIFIC collaboration, B. P. Abbott et al., GW170817: Observation of Gravitational Waves from a Binary Neutron Star Inspiral, Phys. Rev. Lett. 119 (2017) 161101.

[8] V. Connaughton et al., GRB170817A, Gamma Ray Coordinates Network Circular 21506 (2017).

[9] A. Goldstein et al., An Ordinary Short Gamma-Ray Burst with Extraordinary Implications: Fermi-GBM Detection of GRB 170817A., ApJ 848 (Nov., 2017) . 
[10] V. Savchenko et al., Ligo/virgo g298048: Integral detection of a prompt gamma-ray counterpart, GCN 21507 (Aug., 2017) 1-+.

[11] V. Savchenko, C. Ferrigno, E. Kuulkers, A. Bazzano, E. Bozzo, S. Brandt et al., INTEGRAL detection of the first prompt gamma-ray signal coincident with the gravitational wave event GW170817, ApJL, in press (2017) .

[12] W. B. Atwood, A. A. Abdo, M. Ackermann, W. Althouse, B. Anderson, M. Axelsson et al., The Large Area Telescope on the Fermi Gamma-Ray Space Telescope Mission, ApJ 697 (June, 2009) 1071-1102, [0 902 . 1089 ].

[13] G. Vianello, N. Omodei, J. Chiang and S. Digel, Searching for High-energy Gamma-ray Counterparts to Gravitational-wave Sources with Fermi-LAT: A Needle in a Haystack, ApJ 841 (May, 2017) L16, [1607.01793].

[14] M. Ackermann, M. Ajello, A. Albert, B. Anderson, M. Arimoto, W. B. Atwood et al., Fermi-LAT Observations of the LIGO Event GW150914, ApJ 823 (May, 2016) L2, [1602.04488].

[15] J. L. Racusin, E. Burns, A. Goldstein, V. Connaughton, C. A. Wilson-Hodge, P. Jenke et al., Searching the Gamma-Ray Sky for Counterparts to Gravitational Wave Sources: /Fermi GBM and LAT Observations of LVT151012 and GW151226, ApJ 835 (Jan., 2017) 82, [1606.04901].

[16] A. Goldstein, P. Veres, E. Burns, L. Blackburn, M. S. Briggs, N. Christensen et al., Fermi Observations of the LIGO Event GW170104, ApJ 846 (Sept., 2017) L5.

[17] D. A. Coulter, C. D. Kilpatrick, M. R. Siebert, R. J. Foley, M. R. Shappee, J. S. Drout et al., LIGO/Virgo G298048: Potential optical counterpart discovered by Swope telescope, GRB Coordinates Network 21529 (2017)

[18] B. P. Abbott et al., Multi-messenger Observations of a Binary Neutron Star Merge, ApJ in press. (2017) .

[19] B. P. Abbott et al., Gravitational waves and gamma rays from a binary neutron star merger: Gw170817 and grb 170817a., ApJ 828 (2017).

[20] E. Berger, Short-Duration Gamma-Ray Bursts, ARA\&A 52 (Aug., 2014) 43-105, [1311. 2603$].$

[21] P. D’Avanzo, Short gamma-ray bursts: A review, Journal of High Energy Astrophysics 7 (Sept., 2015) 73-80.

[22] J. Granot, A. Panaitescu, P. Kumar and S. E. Woosley, Off-Axis Afterglow Emission from Jetted Gamma-Ray Bursts, ApJ 570 (May, 2002) L61-L64, [astro-ph/0201322].

[23] H. J. van Eerten and A. I. MacFadyen, Observational Implications of Gamma-Ray Burst Afterglow Jet Simulations and Numerical Light Curve Calculations, ApJ 751 (June, 2012) 155, [1105.2485].

[24] E. Troja et al., Ligo/virgo g298048: Chandra monitoring of the x-ray emission from sss17a, GCN 21787 (Sept., 2017) $1-+$.

[25] E. Troja, L. Piro, H. van Eerten, R. T. Wollaeger, M. Im, O. D. Fox et al., Discovery of the X-ray counterpart to the gravitational-wave event GW170817, Nature (Oct., 2017) .

[26] K. P. Mooley et al., Ligo/virgo g298048: Radio detection of sss17a at 3ghz with the vla/jagwar, GCN 21814 (Sept., 2017) 1-+.

[27] Fermi-LAT Collaboration, Fermi-LAT observations of the LIGO/Virgo event GW170817, ArXiv e-prints (Oct., 2017), [1710.05450].

[28] M. Ackermann, M. Ajello, K. Asano, M. Axelsson, L. Baldini, J. Ballet et al., The First Fermi-LAT Gamma-Ray Burst Catalog, ApJS 209 (Nov., 2013) 11, [1303.2908]. 